Angststörungen

\title{
Trends in der psychopharmakologischen Behandlung
}

Dirk Wedekind

Klinik für Psychiatrie und Psychotherapie der Georg-August-Universität Göttingen

psychoneuro 2005; 31 (3): 136-138

Rationalen und Ziele für die Entwicklung neuer Psychopharmaka in der Behandlung von Angststörungen sind neben einer guten Wirksamkeit ein rascher Wirkungsbeginn, gute Verträglichkeit und ausbleibende Toleranz- und Absetzeffekte. Zeitgemäße Entwicklungen zeigen Trends in der Entwicklung selektiver wirksamer serotonerger Substanzen und GABA-Modulatoren, die keine den Benzodiazepinen vergleichbaren unerwünschten Effekte zeigen. Besonders vielversprechende Ansätze zeigen Neuropeptidliganden, die in bestimmte Prozesse bei Angststörungen eingreifen. Hierzu rechnet man im Besonderen Liganden für CCK-4, Substanz-P, Neuropeptid-Y und Sigma-Rezeptoren. Ein wesentlicher zukünftiger Stellenwert ist von Substanzen zu erwarten, die Prozesse im Rahmen der neuroendokrinologischen Stressregulation modulieren, wie CRH-Rezeptorantagonisten und ANP-Agonisten. Viele dieser Strategien sind allerdings noch in frühen Phasen der klinischen Prüfung.

$\mathrm{E}$ ine Kombination von Pharmako- und Psychotherapie gilt heutzutage allgemein als Strategie der Wahl in der Behandlung von Angststörungen. Es liegen mehrere hundert kontrollierte Untersuchungen zur Wirksamkeit unterschiedlicher Substanzen zur Behandlung von Angststörungen vor (1). Hierbei handelt es sich vorrangig um Antidepressiva und Benzodiazepine. Es gibt jedoch einige gute Gründe für die Entwicklung neuer Psychopharmaka für die Angsttherapie. Die heute zur Verfügung stehenden Medikamente aus der Gruppe der serotonerg wirksamen Antidepressiva zeigen zum Beispiel immer noch eine deutliche Verzögerung im Einsetzen der Wirksamkeit. Dies liegt wahrscheinlich an der Zeitspanne, die benötigt wird, um den präsynaptischen, somatodendritischen serotonergen Autorezeptor $\left(5 \mathrm{HT}_{1 \mathrm{~A}}\right) \mathrm{zu}$ desensitivieren (10). Benzodiazepine sind wegen ihres möglichen Abhängigkeits- und To- leranzpotentials ebenfalls problematisch. Andere Substanzen haben dosisabhängig ein nicht akzeptables Nebenwirkungsprofil. handlung von Angststörungen sollte sich neben einer guten Wirksamkeit demnach auch durch einen unmittelbaren Wirkungsbeginn, eine sehr gute Verträglichkeit und durch fehlende Toleranz- und Absetzeffekte auszeichnen.

\section{Serotonerg wirksame Substanzen}

Gerade im Bereich der serotonerg wirksamen Substanzen wurden große Anstrengungen unternommen, Effektivität und Verträglichkeit zu verbessern. Eine der neueren Entwicklungen ist Escitalopram, ein Enantiomer von Citalopram, das bei besserer Verträglichkeit einen rascheren Wirkungsbeginn erwarten lässt. Ein weiterer Entwicklungsweg ist die Herstellung von Pharmaka mit einem spezifi-
Ein ideales Medikament zur Be-

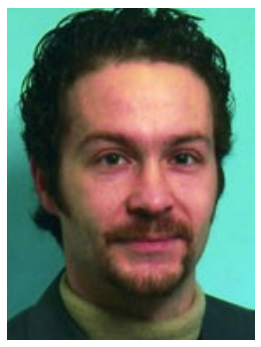

Dirk Wedekind scheren Effekt auf bestimmte Serotonin-(5HT)-Rezeptor-Subtypen, die mit der Pathogenese von Angst in Verbindung gebracht werden. Substanzen mit zusätzlicher oder spezifischer Wirkung auf den $5 \mathrm{HT}_{2}$ - (bzw. $5 \mathrm{HT}_{2 \mathrm{C}^{-}}$) oder den $5 \mathrm{HT}_{1 \mathrm{~A}^{-}}$-Rezeptor sind in letzter Zeit intensiv untersucht worden. Buspiron ist ein selektiver, partieller Agonist am $5 \mathrm{HT}_{1 \mathrm{~A}}$-Rezeptor, der sich als effektiv zur Behandlung der generalisierten Angststörung erwiesen hat. Die $5 \mathrm{HT}_{1 \mathrm{~A}}$-Bindungsstellen werden hierbei heruntergeregelt (16) und ihre Stimulation verstärkt indirekt die serotonerge Aktivität und hat anxiolytische Eigenschaften. Ein weiterer partieller $5 \mathrm{HT}_{1 \mathrm{~A}}$-Agonist, Gepiron, zeigte zumindest Teileffekte bei Panikstörung (13), und eine Studie mit dem Buspiron-Analogon MM199 belegte eine gute anxiolytische Potenz (2). Der vollständige $5 \mathrm{HT}_{1 \mathrm{~A}^{-}}$ Agonist Flesinoxan zeigte allerdings in einer Untersuchung bei Panikstörung sogar eine Verschlechterung der Symptomatik (20).

Zwei Substanzen in fortgeschrittener klinischer Prüfung weisen ein spezifisches $5 \mathrm{HT}_{2 \mathrm{C}}$-antagonistisches Profil auf. Deramciclan und Agomelatin haben einen guten anxiolytischen Effekt, werden aber primär zur Behandlung der Depression untersucht. Eine zusätzliche $5 \mathrm{HT}_{2 \mathrm{C}^{-}}$ antagonistische Komponente bei Serotonin-Wiederaufnahmehemmern könnte das Problem anxiogener Effekte in der frühen Behand- 
lungsphase reduzieren. Mirtazapin zeigte positive Resultate bei der Panikstörung. Der $5 \mathrm{HT}_{3}$-Antagonist Odansetron konnte in bisherigen Studien keine konkreten Effekte zeigen, und schlüssige Ergebnisse für eine ähnliche Substanz, Zatosetron, stehen noch aus.

Inositol ist ein Glukose-Isomer und ein Bestandteil vieler Nahrungsmittel. Inositolphosphat dient als Second-messenger für einige Serotonin-Rezeptortypen aber auch für noradrenerge Rezeptoren. Eine kontrollierte klinische Studie konnte eine gute Wirksamkeit von Inositol bei der Panikstörung zeigen.

\section{GABA-Liganden}

Eine GABA-Dysfunktion ist eine der fundierten Theorien zur Pathogenese von Angststörungen. Bei Benzodiazepinen geht man von einer Verschiebung des Benzodiazepin-Rezeptorkomplexes in eine invers agonistische Richtung aus bzw. einer Defizienz agonistischer, anxiolytischer Liganden. Die angstlösenden Effekte werden über die $\alpha 2$ - und/oder $\alpha 3$-Untereinheit des GABA-A-Rezeptors vermittelt. Partielle GABA-A-Agonisten, die an der $\alpha 2$ - und/oder $\alpha 3$-Untereinheit binden, wie Pagoclon, haben Teileffekte bei der Panikstörung ohne viele der bekannten unerwünschten Begleiteffekte und Absetzschwierigkeiten (15). Nitroflavanoide sind Substanzen mit einer hohen Affinität für den Benzodiazepinrezeptor. Man schreibt ihnen selektive anxiolytische ohne zentralnervös sedierende Effekte zu. Mehrere Substanzen befinden sich aktuell in der frühen Phase klinischer Erprobung. Die bisherigen Ergebnisse lassen aber noch keine konkreten Schlüsse zu.

\section{Kalziumkanal-Liganden}

Gabapentin oder Pregabalin sind Liganden an der Alpha-2-Delta-Untereinheit spannungsabhängiger Kalziumkanäle (21). Gabapentin zeigte in einer kontrollierten Studie positive Ergebnisse bei der Panikstörung (12), während Pregabalin erfolgreich bei der sozialen Angststörung untersucht wurde. Ergebnisse einer Studie bei Panikstörung stehen jedoch noch aus.

\section{- Glutamat-Modulatoren}

Glutamat ist der verbreitetste exzitatorische Transmitter im Gehirn, der in zahlreiche Prozesse involviert ist. Glutamat-Modulatoren sollten entweder postsynaptisch antagonistische (wie LY-326325) oder präsynaptisch agonistische Eigenschaften haben. Daten aus dem Tiermodell geben tatsächlich Hinweise auf hierdurch produzierbare anxiolytische Effekte, Humanstudien fehlen aber bisher.

\section{- Neuropeptide}

Unter den Neuropeptiden, die mit Angst und im Besonderen mit Panik in Verbindung gebracht werden, ist Cholecystokinin das am besten untersuchte. Das Tetrapeptid (CCK-4) und das synthetische Pentapeptid Pentagastrin können bei Gesunden und in bedeutend geringeren Dosierungen bei Menschen mit einer Panikstörung Panikattacken hervorrufen. CCK-4-Bindungsstellen finden sich in verschiedenen Hirnregionen, besonders aber in limbischen Strukturen wie Amygdala, Hippocampus und dem noradrenergen Kernzentrum, dem Locus coeruleus im Hirnstamm. Die Tatsache, dass CCK-4-Provokationstests nicht nur Angstgefühle, sondern auch körperliche Angstsymptome ohne herausragende psychische Angst bedingen können, weist auf einen Einfluss auf unterschiedliche Anteile des zentralnervösen „Angstnetzwerks“ hin. CCK-4-Antagonisten konnten in den bisherigen Untersuchungen jedoch noch keine wesentlichen Effekte in der Behandlung, z.B. der Panikstörung zeigen, wenngleich diese aber durch CCK-4 induzierte Panikattacken unterdrücken konnten (19).

Ein weiteres interessantes Neuropeptid ist Substanz-P, ein Neurokinin-1-Rezeptoragonist. Substanz-P spielt eine wichtige Rolle bei Angst und Panik ähnlichem Verhalten und ist häufig in noradrenergen und serotonergen Zellen kolokalisiert (14). Erste Untersuchungen mit Antagonisten wie MK869 bei Angst im Rahmen einer Depression zeigten vielversprechende Ergebnisse (7) und Studien bei Panikstörung werden unternommen.
Neuropeptid-Y ist ein aus 36 Aminosäuren bestehendes neuroaktives Peptid, das an der Regulation zentralnervöser noradrenerger Aktivität beteiligt ist und eine hohe Dichte von Bindungsstellen im Hypothalamus aufweist. Noradrenalin ist sicherlich in der Angstentstehung beteiligt. Obwohl die meisten noradrenerg wirksamen Antidepressiva keine relevante Wirksamkeit bei Angststörungen zeigten, gibt es Hinweise für die Effektivität des selektiven Noradrenalin-Wiederaufnahmehemmers Reboxetin. Ein Neuropeptid-Y-Ligand könnte daher eine interessante Substanz sein, obwohl klinische Studien bisher fehlen und auch keine oral verabreichbare synthetische Substanz zur Verfügung steht.

Sigmarezeptoren finden sich in besonders hoher Konzentration in verschiedenen Bereichen des Zwischenhirns. Man schreibt ihnen eine neuromodulatorische Funktion auf noradrenerge und auch serotonerge Zellkernareale zu. Verschiedene Substanzen befinden sich in der klinischen Erprobung. Möglicherweise zeichnen sich Sigma-Rezeptorliganden durch ein sehr günstiges Nebenwirkungsprofil aus. Aussagekräftige Ergebnisse liegen aber noch nicht vor.

\section{Inhibitoren der Steroid- Synthese}

Viele psychiatrische Störungen gehen mit einer lang andauernden Hyperaktivität der HypothalamusHypophysen-NebennierenrindenAchse und einer Hypersekretion von Kortisol einher. Substanzen, die die Aktivität dieses neuroendokrinen Prozesses modulieren, sind in den letzten Jahren intensiv beforscht worden. Das Mykostatikum Ketokonazol, ein Steroid-Synthese-Blocker, zeigte zum Beispiel eine Plazebo überlegene Wirkung in der Behandlung depressiver Patienten mit einem Hyperkortisolismus (22). Aber ebenso wie beim Progesteron-Antagonist Mifepriston („Abtreibungspille“) liegen noch keine Studien bei Angststörungen vor (9). Auch Metyrapon, ein anderer Glukokortikoid-Synthese-Hemmer, erwies sich in Tiermodellen zur Depression als effektiv (3) und auch in einer kleineren kontrollierten Depressionsstudie (11). 
Corticotropin-Releasing-Hormon (CRH) bedingt unterschiedliche Korrelate von Stress, Angst und Panik im zentralen Nervensystem. Eine Regulierung der CRH-Aktivität ist möglicherweise ein lohnenswertes Ziel der pharmakologischen Forschung bei Angststörungen. CRHAntagonisten können nach intraventrikulärer Applikation im Tierversuch Angst- und Stresssymptome vermindern (8). Für klinische Untersuchungen stellen möglicherweise Substanzen, die antagonistisch am CRH- oder GlukokortikoidRezeptor wirken, einen wesentlichen Fortschritt da. Das gleiche gilt für Agonisten am Rezeptor für atriales natriuretisches Peptid (ANP), das die Funktion der HypothalamusHypophysen-Nebennieren-Achse an verschiedenen Stellen moduliert. ANP hat anxiolytische Effekte im Tierversuch (17) und ANP-Agonisten können bei Panikpatienten wie auch bei Gesunden Panikattacken durch CCK-4 Provokation vermeiden (18).

Am weitesten fortgeschritten sind Untersuchungen mit verschiedenen CRH-1-Rezeptorantagonisten. Im Tierversuch konnten sowohl Hyperkortisolismus und ängstliches Verhalten bei speziellen Rattenstämmen reduziert werden $(5,6)$. Eine offene Humanstudie mit einem CRH1-Antagonisten konnte bei Major Depression Angst und Depressivität reduzieren (4). Ein weiteres interessantes Konzept wäre die Kombination einer solchen Substanz mit einem serotonerg wirksamen Antidepressivum, um die Wirklatenz zu verkürzen.

\section{Fazit}

Von der ersten Phase der klinischen Erprobung bis zur Zulassung vergehen üblicherweise viele Jahre. Von den vielen der genannten vielversprechenden Substanzen wird sicherlich nur ein Teil in den Handel kommen. Die wichtigsten Entwicklungen für die Zukunft anxiolytischer Pharmakotherapie sind Neuropeptid-Rezeptor-Liganden und spezifische Modulatoren am GABA-A-Benzodiazepin-Rezeptorkomplex und des serotonergen Systems.
Trends in the psychopharmacological treatment of anxiety disorders The rationale for, and the aims of, the development of new psychopharmacological substances for the treatment of anxiety disorders are, in addition to effectiveness, a rapid onset of effect, good tolerability and an absence of tolerance and discontinuation effects. Modern developments reveal trends in the development of selectively effective serotonergic substances and GABA modulators, which show none of the undesired effects comparable with those seen with benzodiazepines. Particularly promising approaches are the neuropeptide ligands that interfere with certain processes in anxiety disorders. These substances include in particular ligands for CCK-4, substance $P$, neuropeptide $Y$, and sigma receptors. $A$ major future impact is to be expected from substances that modulate processes within the framework of neuroendocrinal stress regulation, such as $\mathrm{CRH}$ receptor antagonists and ANP agonists. It must be noted, however, that many of these strategies are still in the early stages of clinical testing.

\section{Key Words}

therapeutic interventions - anxiety panic disorders - new strategies

\section{Literatur}

1. Bandelow B et al. WFSBP Task force on treatment guidelines for the pharmacological treatment of anxiety - obsessive compulsive disorders and posttraumatic stress disorder. World J Biol Psychiatry 2002; 3: 171-199 2. Deren-Wesolek A, Tatarczynska E, Chojnacka-Wojcik $E$. The novel buspirone analogue, 8-(4-(2-(1,2,3,4-tetrahydroisoquinolinyl)(butyl)-8-azaspiro (4.5) decane-7,9dione, with anxiolytic-like and antidepressant-like effects in rats. J Psychopharmacol 1998; 12(4): 380-384

3. Healy DG et al. Metyrapone displays antidepressant-like properties in preclinical paradigms. Psychopharmacology 1999; 145(3): 303-308

4. Holsboer F. The rationale for corticotropin-releasing hormone receptor (CRH-R) antagonists to treat depression and anxiety. J Psychiatr Res 1999; 33: 181-214

5. Keck ME, Holsboer F. Hyperactivity of $\mathrm{CRH}$ neuronal circuits as a target for therapeutic interventions in affective disorders. Peptides 2001; 22(5): 835-844

6 . Keck ME et al. The high-affinity nonpeptide CRH1 receptor antagonist R121919 attenuates stress-induced alterations in plasma oxytocin, prolactin, and testosterone secretion in rats. Pharmacopsychiatry 2003; 36(1): 27-31
7. Kramer MS et al. Distinct mechanism for antidepressant activity by blockade of central substance $\mathrm{P}$ receptors. Science 1998; 281(5383): 1640-1645

8. Liebsch $\mathrm{F}$ et al. Differential behavioural effects of chronic infusion of $\mathrm{CRH} 1$ and $\mathrm{CRH} 2$ receptor antisense oligonucleotides into the rat brain. J Psychiatry Res 1999; 33: 153-163 9. Murphy BE, Filipini D, Ghadirian AM. Possible use of glucocorticoid receptor antagonists in the treatment of major depression: preliminary results using RU 486. J Psychiatry Neurosci 1993; 18(5): 209-213

10. Nutt DJ et al. Mechanisms of action of selective serotonin reuptake inhibitors in the treatment of psychiatric disorders. Eur Neuropsychopharmacol 1999; 9 (suppl. 3): 81-86 11. O'Dwyer AM et al. Teatment of amjor depression with metyrapone and hydrocortisone. J Affect Disord 1995; 33(2): 123-128

12. Pande AC et al. Placebo-controlled of gabapentin treatment of panic disorder. J Clin Psychopharmacol 2000; 20:467-471

13. Pecknold JC et al. Gepirone and the treatment of panic disorder: an open study. J Clin Psychopharmacol 1993; 13: 145-149

14. Pelletier G, Steinbusch HW, Verhofstad AA. Immunoreactive substance $P$ and serotonin present in the same dense-core vesicles. Nature 1981; 293(5827): 71-72

15. Sandford JJ et al. Crossover trial of pagoclone and placebo in patients with DSM-IV panic disorder. J Psychopharmacol 2001; 15(3): 205-208

16. Sargent PA et al. Brain serotonin1A receptor binding measured by positron emission tomography with (11C)WAY-100635: effects of depression and antidepressant treatment. Arch Gen Psychiatry 2000; 57(2): 174-180

17. Ströhle A et al. Central and peripheral administration of atriopeptin is anxiolytic in rats. Neuroendocrinology 1997; 65: 210-215

18. Ströhle A et al. Anxiolytic activity of atrial natriuretic peptide in patients with panic disorder. Am J Psychiatry 2001; 158(9): 1514-1514

19. Van Megen $\mathrm{HJ}$ et al. The cholecystokinin$\mathrm{B}$ receptor antagonist $\mathrm{Cl}-988$ failed to affect CCK-4 induced symptoms in panic disorder patients. Psychopharmacology 1997; 129 : 243-248

20. Van Vliet IM, Westenberg HG, Den Boer JA. Effects of the $5 \mathrm{HT} 1 \mathrm{~A}$ receptor agonist flesonoxan in panic disorder. Psychopharmacology 1996; 127: 174-180

21. Wedekind D, Bandelow B. Die Alpha-2Delta-Untereinheit des spannungsabhängigen Kalziumkanals als pharmakologisches Ziel in der Psychiatrie und Neurologie. Nervenarzt (eingereicht)

22. Wolkowitz OM et al. Antiglucocorticoid treatment of depression: double-blind ketokonazole. Biol Psychiatry 1999; 45(8): 1070-1074

\section{Korrespondenzadresse:}

Dr. med. Dirk Wedekind, MSc Klinik für Psychiarie und Psychotherapie der Georg-August-Universität Göttingen Von-Siebold-Straße 5

37075 Göttingen

dwedeki1@gwdg.de 